\title{
Feedback function for capillary refilling time measurement device
}

\author{
Masayoshi Shinozaki ${ }^{1}$, Taka-aki Nakada ${ }^{2 *}$, Rui Kawaguchi ${ }^{2}$, Yuichiro Yoshimura', Toshiya Nakaguchi', \\ Hideaki Haneishi ${ }^{1}$ and Shigeto Oda ${ }^{2}$
}

This comment refers to the article available at https://doi.org/10.1186/s13054-018-2295-3.

Capillary refilling time (CRT) is an important indicator of microcirculation $[1,2]$. To develop a CRT measurement device, the optimal strength and time for pressing the nail bed were investigated [3]. However, whether examiners can precisely achieve optimal strength and time remains unknown. Thus, the requirements for a CRT measurement device have not yet been fully elucidated. We developed a portable CRT measurement device with a feedback function to achieve optimal strength and time (Fig. 1) and tested a hypothesis that the feedback function is the key for satisfying the measurement conditions of the CRT device.

The CRT was measured by 20 examiners using the developed device with and without a feedback function. According to a previous report [3], the target strength of $5 \mathrm{~N}$ and time of $5 \mathrm{~s}$ were obtained (Additional file 1). The pressing strength and time during the CRT measurement were evaluated.

A significant difference was found in the pressing strength and time between the CRT measurement using the device with and without a feedback function (strength: $P<0.001$; time: $P<0.01$ ). The feedback function significantly reduced the intra-examiner variance in the pressing strength and time (strength: $P<0.001$; time: $P<0.001$ ) (Fig. 2). In all measurements without the feedback function, $41 \%$ of the pressing strength was outside the required strength range. In contrast, in the CRT measurements with the feedback function, $100 \%$ of the pressing strength was successfully achieved within the target range. The pressing time with the feedback function achieved the target time in all measurements, whereas $12 \%$ of the measurements without the feedback function exhibited insufficient pressing time. In total, $49 \%$ of the measurements without the feedback function failed to satisfy the required conditions.

Compared with the high failure rate of the CRT measurements without the feedback function, the feedback function, which served as a guide for the pressing strength and time, achieved complete success in fulfilling the required measurement conditions of the CRT measurement using the portable device. The critical issue of the CRT measurement is the intra-observer difference [4]. Personal work experience and training have been suggested to possibly help improve the accuracy of CRT measurements [5]. Evidently, in the present study, the feedback function

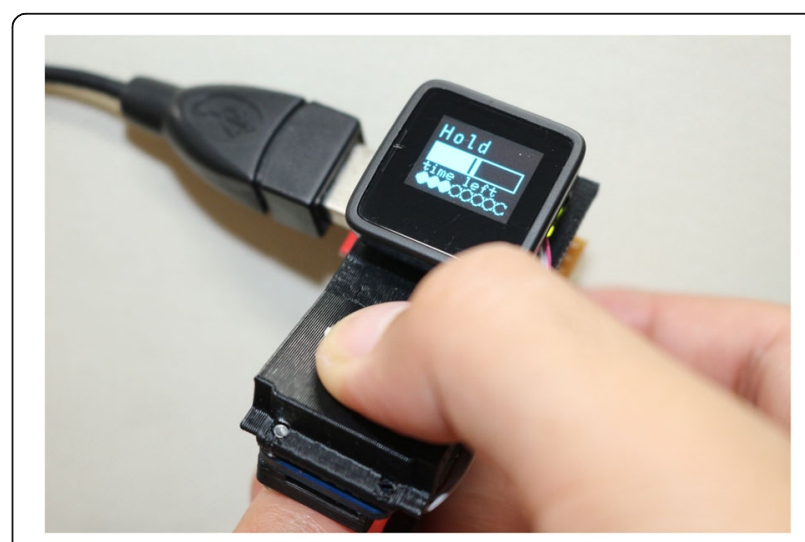

Fig. 1 Photograph of the developed device. The feedback function is shown in an OLED display

\footnotetext{
* Correspondence: taka.nakada@nifty.com

${ }^{2}$ Department of Emergency and Critical Care Medicine, Chiba University

Graduate School of Medicine, 1-8-1 Inohana, Chuo, Chiba 260-8677, Japan

Full list of author information is available at the end of the article
}

(c) The Author(s). 2019 Open Access This article is distributed under the terms of the Creative Commons Attribution 4.0 International License (http://creativecommons.org/licenses/by/4.0/), which permits unrestricted use, distribution, and

reproduction in any medium, provided you give appropriate credit to the original author(s) and the source, provide a link to the Creative Commons license, and indicate if changes were made. The Creative Commons Public Domain Dedication waiver (http://creativecommons.org/publicdomain/zero/1.0/) applies to the data made available in this article, unless otherwise stated. 


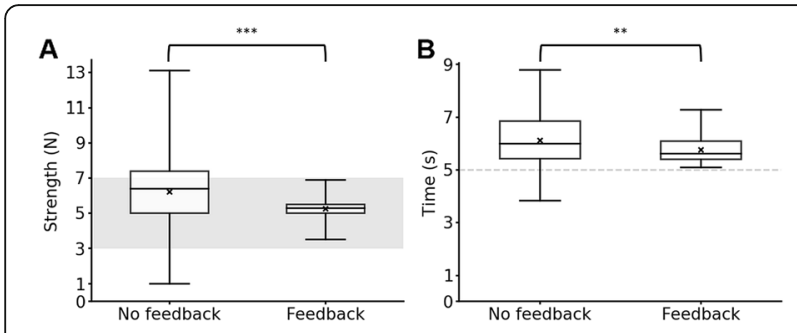

Fig. 2 a, b Pressing strength and time in the CRT measurement using the developed device without and with feedback function. Panel a Pressing strength. Panel b Pressing time. A significant difference was observed in the pressing strength and time between the CRT measurements using the portable CRT device with and without a feedback function (Mann-Whitney $U$ test - strength: $P<0.001$; time: $P<0.01)$. The feedback function significantly reduced the intra-examiner variance in the pressing strength and time ( $F$ test - strength: $P<0.001$; time: $P<0.001$ ). The median and minimum and maximum interquartile ranges are shown

significantly reduced the intra-examiner variance. Thus, this device would be a smart solution for intra-observer difference regardless of personal work experience and without training. Further development of portable CRT measurement devices with feedback functions may contribute to achieve precise CRT measurements to monitor microcirculation in clinical settings.

\section{Additional file}

Additional file 1: Methods and supplemental data. Figure E1. Appearance of the developed device and display of the feedback function. (a) Front image. (b) Side image. (c) Oblique image with a finger. (d)-(h) Display changes in the feedback function. (d) Ready for measurement. (e) The filled range indicates the strength applied to the nail bed, and the vertical line shows the target strength. This display indicates insufficient pressing strength. (f) The display shown after the target strength is applied. The filled circle indicates the time to press, and the blue circle indicates the remaining time to press the nail bed. (g) The display tells the examiner to release the compression. (h) The display is shown after the release of the nail bed, which shows the interval until the next measurement. (PDF $1307 \mathrm{~kb}$ )

\section{Availability of data and materials}

The datasets used and analyzed during our study are available from the corresponding author upon reasonable request.

\section{Ethics approval and consent to participate}

The study participants were informed of the experiment, and permissions were obtained in a consent form before the measurements. All procedures in this study were approved by the Ethical Review Board of the Chiba University Graduate School of Medicine.

\section{Consent for publications}

A written informed consent for publication was obtained.

\section{Competing interests}

The authors declare that they have no competing interests. Chiba University has filed a provisional patent application covering the aspects of this manuscript. TN, TN, HH, and SO are listed as inventors.

\section{Author details}

${ }^{1}$ Center for Frontier Medical Engineering, Chiba University, 1-33, Yayoicho, Inage, Chiba 263-8522, Japan. ${ }^{2}$ Department of Emergency and Critical Care Medicine, Chiba University Graduate School of Medicine, 1-8-1 Inohana, Chuo, Chiba 260-8677, Japan.

Received: 25 July 2019 Accepted: 16 August 2019

Published online: 03 September 2019

\section{References}

1. Ait-Oufella H, Bige N, Boelle PY, Pichereau C, Alves M, Bertinchamp R, Baudel JL, Galbois A, Maury E, Guidet B. Capillary refill time exploration during septic shock. Intensive Care Med. 2014;40:958-64.

2. De Backer D, Ospina-Tascon G, Salgado D, Favory R, Creteur J, Vincent JL. Monitoring the microcirculation in the critically ill patient: current methods and future approaches. Intensive Care Med. 2010;36:1813-25.

3. Kawaguchi R, Nakada TA, Oshima T, Shinozaki M, Nakaguchi T, Haneishi H, Oda S. Optimal pressing strength and time for capillary refilling time. Crit Care. 2019;23:4.

4. Hariri G, Joffre J, Leblanc G, Bonsey M, Lavillegrand JR, Urbina T, Guidet B, Maury E, Bakker J, Ait-Oufella H. Narrative review: clinical assessment of peripheral tissue perfusion in septic shock. Ann Intensive Care. 2019;9:37.

5. Shinozaki K, Jacobson LS, Saeki K, Kobayashi N, Weisner S, Falotico JM, Li T, Kim J, Lampe JW, Becker LB. Does training level affect the accuracy of visual assessment of capillary refill time? Crit Care. 2019;23:157.

\section{Publisher's Note}

Springer Nature remains neutral with regard to jurisdictional claims in published maps and institutional affiliations.

\begin{abstract}
Authors' contributions
RK and TN contributed to the study conceptualization and design, interpretation of data, statistical analysis, and drafting and critical revision of the manuscript for important intellectual content. MS, YY, and TN contributed to the device development, study conceptualization and design, data acquisition, data analysis and interpretation, statistical analysis, and drafting and critical revision of the manuscript for important intellectual content. $\mathrm{HH}$ and $\mathrm{SO}$ contributed to the study conceptualization and critical revision of the manuscript for important intellectual content. All authors have read and approved the final manuscript.
\end{abstract}

\section{Funding}

This work was supported by AMED (\#JPhe1502001) and JSPS KAKENHI Grant Number 18K16530. The funder had no role in the study design, experiments, collection, analyses, interpretation of data, writing of the manuscript, or decision to submit the manuscript for publication. 